\title{
Study of $B$ meson decays to baryons at BABAR
}

\author{
Roland Waldi* \\ Universität Rostock \\ E-mail: roland.waldi@uni-rostock.de \\ for the BABAR Collaboration
}

\begin{abstract}
Baryonic decays account for about $7 \%$ of the $B$-meson width, and have been studied in recent years by the B factories. These studies reveal properties of hadronization at low $q^{2}$, such as $s \bar{s}$ suppression known from jet fragmentation, and phase space relations between the baryon and antibaryon. The measurement and comparison of exclusive branching fractions of $B$ decays to baryons as well as studies on the dynamics of the decay allow better understanding of these properties. We present the most recent measurements of $B$-meson decays with two or four baryons in the final state performed with the BABAR detector.
\end{abstract}

The European Physical Society Conference on High Energy Physics - EPS-HEP2013 18-24 July 2013

Stockholm, Sweden

${ }^{*}$ Speaker. 


\section{Introduction}

Baryons in the final state occur in pairs due to baryon number conservation, hence the minimum mass of a meson that can decay into baryons is above $1.9 \mathrm{GeV}$. There are only very few examples at masses below the $B$ meson: $D_{s}^{+} \rightarrow p \bar{n}$ and $J / \psi \rightarrow$ baryons. But the weakly decaying $B$ mesons with a mass of $5.28 \mathrm{GeV}$ are far above the threshold and allow a multitude of different channels, including, in principle, even final states with four baryons.

Inclusive studies at ARGUS [1] have shown that $(6.8 \pm 0.6) \%$ of all $B$ decays have baryons in their final state. Exclusive channels have been investigated by CLEO and the B Factories BABAR and Belle, adding up to $(0.53 \pm 0.06) \%\left(B^{0}\right)$ and $(0.85 \pm 0.15) \%\left(B^{+}\right)$, respectively [2]. This is still a small fraction of the total, but enough to show some salient features: two-body branching fractions are much smaller than similar three-body rates, and there is still a moderate increase in branching fraction to higher multiplicities. Also, most multibody channels show a more or less pronounced enhancement at the threshold of the baryon-antibaryon invariant mass.

Understanding the production of baryons in $B$ decays is also important on the route to an understanding of the baryon-antibaryon asymmetry of the universe: $B$ mesons exhibit the largest $\mathrm{CP}$ asymmetries seen so far, and they belong to the small group of mesons that can produce baryons. Whether that leads to an explanation of the baryon-antibaryon asymmetry depends on the missing link: baryon number violation, that has not yet been observed in any process.

\section{Recent BABAR results on $\bar{B}^{0} \rightarrow \Lambda_{c} \bar{p} \pi^{+} \pi^{-}$}

One of the high-statistics channels is the decay $\bar{B}^{0} \rightarrow \Lambda_{c} \bar{p} \pi^{+} \pi^{-}$, with a branching fraction [3]

$$
\mathscr{B}\left(\bar{B}^{0} \rightarrow \Lambda_{c} \bar{p} \pi^{+} \pi^{-}\right)=(12.3 \pm 0.5 \pm 0.7 \pm 3.2) \cdot 10^{-4}
$$

where the errors are statistical, systematic and from the uncertainty in the absolute $\Lambda_{c}$ branching fraction scale, $\mathscr{B}\left(\Lambda_{c} \rightarrow p K^{-} \pi^{+}\right)=(5.0 \pm 1.3) \%$. The most prominent three-body subchannels are

$$
\begin{aligned}
\mathscr{B}\left(\bar{B}^{0} \rightarrow \Sigma_{c}(2455)^{++} \bar{p} \pi^{-}\right) & =(2.13 \pm 0.10 \pm 0.10 \pm 0.55) \cdot 10^{-4} \\
\mathscr{B}\left(\bar{B}^{0} \rightarrow \Sigma_{c}(2520)^{++} \bar{p} \pi^{-}\right) & =(1.15 \pm 0.10 \pm 0.05 \pm 0.30) \cdot 10^{-4} \\
\mathscr{B}\left(\bar{B}^{0} \rightarrow \Sigma_{c}(2455)^{0} \bar{p} \pi^{+}\right) & =(0.91 \pm 0.07 \pm 0.04 \pm 0.24) \cdot 10^{-4} \\
\mathscr{B}\left(\bar{B}^{0} \rightarrow \Sigma_{c}(2520)^{0} \bar{p} \pi^{+}\right) & =(0.22 \pm 0.07 \pm 0.01 \pm 0.06) \cdot 10^{-4}
\end{aligned}
$$

where the last one cannot be significantly established.

Although this four-body branching fraction is larger than the ones of similar three-body decays like $\bar{B}^{0} \rightarrow \Lambda_{c} \bar{p} \pi^{0}$ or $B^{-} \rightarrow \Lambda_{c} \bar{p} \pi^{-}$, this does not prove an increase with multiplicity because a large fraction corresponds in fact to subsequent decays of three-body final states. The ground-state $\Sigma_{c}^{++}$ and $\Sigma_{c}^{0}$ baryons already account for about $36 \%$, and other resonances like $\Sigma_{c}(2800), \rho^{0}$ and $\Delta$ are clearly visible in the invariant two-body mass distributions of these decays [3].

\section{The threshold enhancement}

An enhancement at the threshold of the invariant baryon-antibaryon mass has been observed in many $B$ meson decays to baryons, prominent examples are the $\Lambda_{c}^{+} \bar{p} \pi^{-}$and $D^{0} p \bar{p}$ final states (Figs. 1a and b). It is also present in the $\Sigma_{c}^{++} \bar{p} \pi^{-}$final state (Fig. 1c), but less pronounced. 
a)

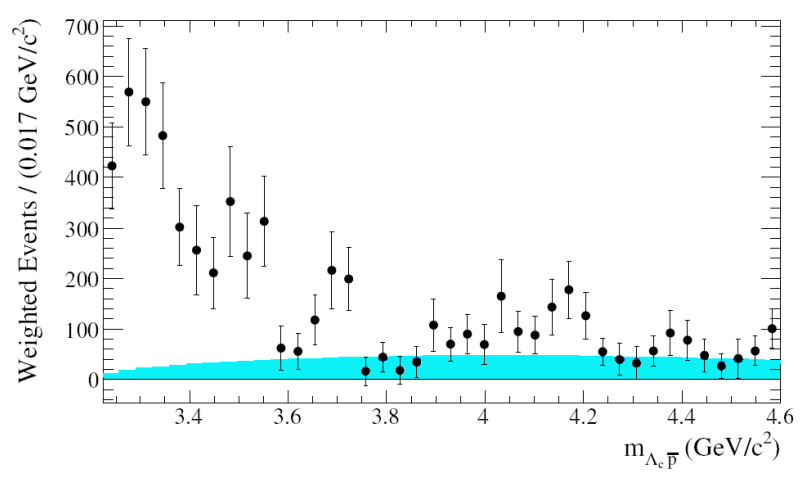

b)

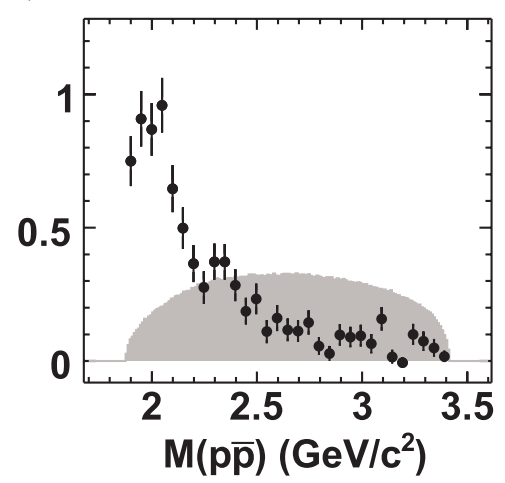

c)

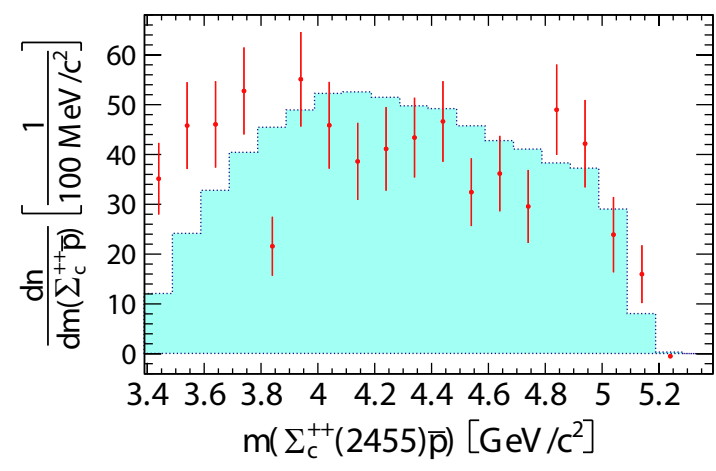

d)

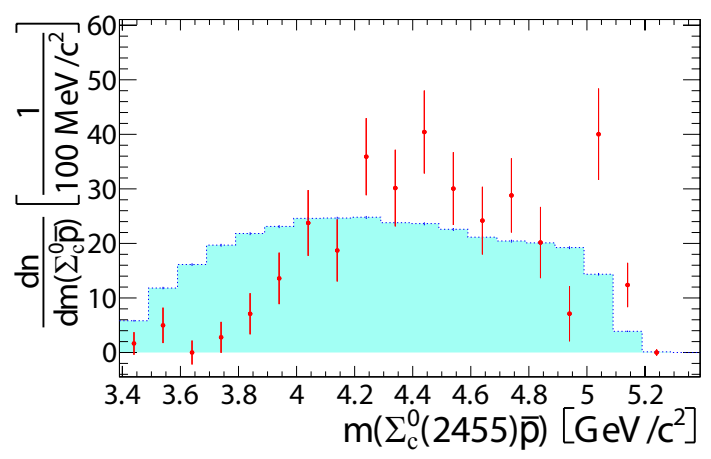

Figure 1: Invariant baryon antibaryon mass distribution in the decay (a) $B^{-} \rightarrow \Lambda_{c} \bar{p} \pi^{-}$[5], (b) $\bar{B}^{0} \rightarrow$ $\Sigma_{c}^{++} \bar{p} \pi^{-}$[6], (c) $\bar{B}^{0} \rightarrow \Sigma_{c}^{++} \bar{p} \pi^{-}$, and (d) $\bar{B}^{0} \rightarrow \Sigma_{c}^{0} \bar{p} \pi^{+}$(points with error bars) compared to a three-body phase space distribution (filled histograms) [3].

I want to elaborate a little bit on this feature using the example in Figs. 1a and d. Examples of Feynman diagrams (where gluons are omitted) are shown in Fig. 2. The colour suppressed or "internal $W$ " (operator $O_{2}$ ) diagram in Fig. $2 \mathrm{~b}$ as well as other tree and penguin diagrams have one feature in common: if we omit the quarks created from the vacuum, they represent decays to meson pairs, and the extra $q \bar{q}$ pairs from the vacuum form the baryon-antibaryon pair from one of these mesons. In a phenomenological pole model, they emerge through a meson pole with a pole mass below threshold, and therefore lead to a steeply falling matrix element at the threshold. In a more QCD-motivated picture, the gluons needed to produce these extra pairs tend to have low $q^{2}$ due to the running of $\alpha_{S}$ and the propagator $\propto 1 / q^{2}$. A typical example is the decay $B^{-} \rightarrow \Lambda_{c}^{+} \bar{p} \pi^{-}$ $[4,5]$ shown in Fig. 1a, while the decay $\bar{B}^{0} \rightarrow \Sigma_{c}(2455)^{++} \bar{p} \pi^{-}$in Fig. 1c has both a mesonmeson diagram (colour favoured, $O_{1}$ ) and a diquark-antidiquark diagram and therefore only a small enhancement.

A counterexample is the decay $\bar{B}^{0} \rightarrow \Sigma_{c}^{0} \bar{p} \pi^{+}$shown in Fig. 1d where the threshold region is hardly populated. Indeed, all diagrams like the example in Fig. $2 \mathrm{~d}$ would not lead to meson pairs, but rather to a diquark-antidiquark state. In a pole model, only a baryon pole could be assumed to create a baryon meson pair, and the $q \bar{q}$ pairs must be created by hard gluons. The latter is particularly the case with two-body decays, where only one $q \bar{q}$ pair with high $q^{2}$ is produced. This explains the substantial suppression of two-body decays. 
a)

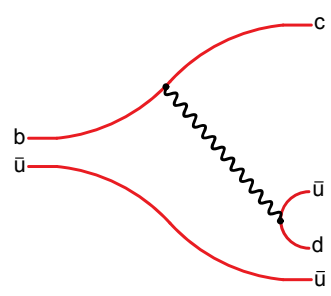

b)

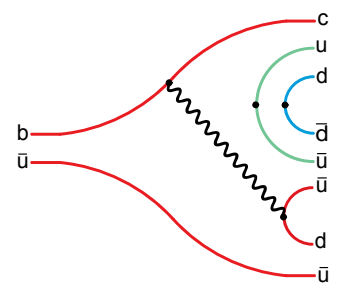

c)

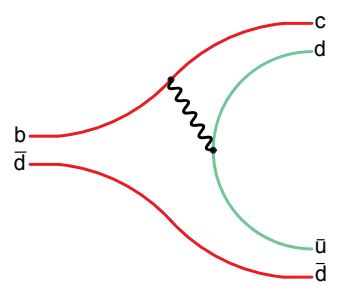

d)

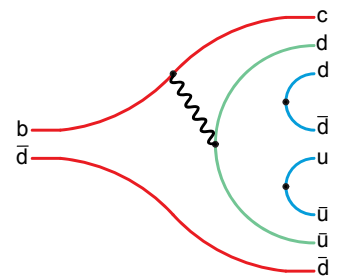

Figure 2: Spectator diagrams (corresponding to operator $\mathrm{O}_{2}$ ) illustrating the basic picture for a baryon antibaryon threshold enhancement: (b) shows one diagram for $B^{-} \rightarrow \Lambda_{c}^{+} \bar{p} \pi^{-}$with preferentially low baryon antibaryon mass related to meson pair diagram (a), and (d) for $\bar{B}^{0} \rightarrow \Sigma_{c}^{0} \bar{p} \pi^{+}$with preferentially high baryon antibaryon mass related to diquark pair diagram (c). The colours indicate allowed combinations of colour quantum numbers of the quarks.

While theorists have suggested explanations along these lines [7], a rigorous calculation is still missing.

\section{Recent BABAR results on $\bar{B}^{0} \rightarrow \Lambda_{c} \bar{p} p \bar{p}$}

The final state with four baryons has a very small phase space: about $1 / 1450$ th of that for $\bar{B}^{0} \rightarrow \Lambda_{c} \bar{p} \pi^{+} \pi^{-}$. However, we just learned that a threshold enhancement in the baryon-antibaryon mass leads to a clustering in the available phase space, i.e., only a small portion of the four-body phase space is actually used by final states with baryons. This fact may reduce or even cancel the suppression due to the small phase space, which can be checked via the four-baryon final state.
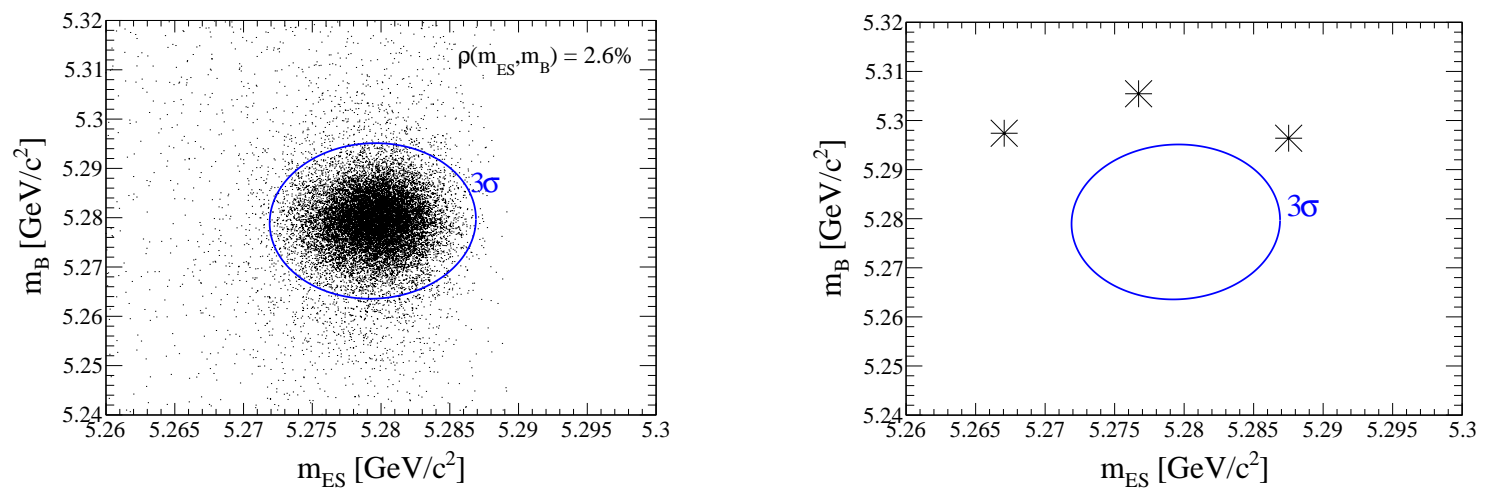

Figure 3: Three-sigma signal region populated by simulated events (a) and completely empty for data (b).

BABAR finds [8], however, only three background events and no event in the signal region (Fig. 3). This translates into an upper limit

$$
\mathscr{B}\left(\bar{B}^{0} \rightarrow \Lambda_{c} \bar{p} p \bar{p}\right) \cdot(1 \pm 0.26)<2.8 \cdot 10^{-6} \text { at } 90 \% \mathrm{CL}
$$

where the factor $(1 \pm 0.26)$ describes the scaling factor for absolute $\Lambda_{c}$ branching fractions

$$
\frac{\mathscr{B}\left(\Lambda_{c} \rightarrow p K^{-} \pi^{+}\right)}{5.0 \%}=1.00 \pm 0.26
$$


that is an external input and subject to change in the future. This factor cancels when the branching fraction is compared to other final states with a $\Lambda_{c}$ baryon. If we take the four-body component of $\bar{B}^{0} \rightarrow \Lambda_{c} \bar{p} \pi^{+} \pi^{-}$to be about one half of the total, this limit translates into

$$
\frac{\mathscr{B}\left(\bar{B}^{0} \rightarrow \Lambda_{c} \bar{p} p \bar{p}\right)}{\mathscr{B}\left(\bar{B}^{0} \rightarrow \Lambda_{c} \bar{p} \pi^{+} \pi_{4 \text { body }}^{-}\right)} \lesssim \frac{1}{200}
$$

which is still one order of magnitude off the pure phase space factor of $1 / 1450$. Therefore, we can only exclude a large enhancement from the low-mass baryon antibaryon production mechanism.

\section{Recent BABAR results on $\bar{B}^{0} \rightarrow D^{0} \Lambda \bar{\Lambda}$}

The decay diagrams for $\bar{B}^{0} \rightarrow D^{0} \Lambda \bar{\Lambda}$ are the same as for $\bar{B}^{0} \rightarrow D^{0} p \bar{p}$, with a $u \bar{u}$ pair created from the vacuum replaced by an $s \bar{s}$ pair. If we assume that the hadronization process from the weak decay to $c \bar{d} \bar{u} d$ proceeds in the same way as quark fragmentation in jets, we expect a suppression of $s \bar{s}$ pair production by about a factor $1 / 3$, and democratic formation of $\Lambda$ and $\Sigma^{0}$ from an $u d s$ colour singlet state. This assumes no particular isospin dominance in the $c \bar{d} \bar{u} d$ system. Combining these assumptions, we expect a ratio ${ }^{1}$

$$
\frac{\mathscr{B}\left(\bar{B}^{0} \rightarrow D^{0} \Lambda \bar{\Lambda}\right)}{\mathscr{B}\left(\bar{B}^{0} \rightarrow D^{0} p \bar{p}\right)} \approx \frac{1}{12}
$$

and

$$
\frac{\mathscr{B}\left(\bar{B}^{0} \rightarrow D^{0} \Sigma^{0} \bar{\Lambda}\right)+\mathscr{B}\left(\bar{B}^{0} \rightarrow D^{0} \Lambda \bar{\Sigma}^{0}\right)}{\mathscr{B}\left(\bar{B}^{0} \rightarrow D^{0} \Lambda \bar{\Lambda}\right)} \approx 2
$$

The combined analysis [9] using three $D^{0}$ decay channels to $K^{-} \pi^{+}, K^{-} \pi^{+} \pi^{0}$ and $K^{-} \pi^{+} \pi^{+} \pi^{-}$ results in a signal with $3.4 \sigma$ significance, corresponding to

$$
\mathscr{B}\left(\bar{B}^{0} \rightarrow D^{0} \Lambda \bar{\Lambda}\right)=\left(9.8 \pm{ }_{2.6}^{2.9} \pm 1.9\right) \cdot 10^{-6}
$$

which can be translated into

$$
\frac{\mathscr{B}\left(\bar{B}^{0} \rightarrow D^{0} \Lambda \bar{\Lambda}\right)}{\mathscr{B}\left(\bar{B}^{0} \rightarrow D^{0} p \bar{p}\right)} \approx \frac{1}{10.6 \pm 3.7}
$$

using the world average $\mathscr{B}\left(\bar{B}^{0} \rightarrow D^{0} p \bar{p}\right)=(1.04 \pm 0.07) \cdot 10^{-4}$ [2]. This is consistent with the fragmentation model, while there is some tension with the prediction of $\mathscr{B}\left(\bar{B}^{0} \rightarrow D^{0} \Lambda \bar{\Lambda}\right)=(2.3 \pm$ $0.8) \cdot 10^{-6}$ in Ref. [10]. This discrepancy is compensated by much larger predictions for $\bar{B}^{0} \rightarrow$ $D^{0}+\left(\Sigma^{0} \bar{\Lambda}, \Lambda \bar{\Sigma}^{0}\right.$ or $\left.\Sigma^{0} \bar{\Sigma}^{0}\right)$ in a related paper [11].

In the BABAR analysis, a significant background was from $\Sigma^{0} \rightarrow \gamma \Lambda$ decays. From the background contribution in the fit we obtain a rough guess of

$$
\frac{\mathscr{B}\left(\bar{B}^{0} \rightarrow D^{0} \Sigma^{0} \bar{\Lambda}\right)+\mathscr{B}\left(\bar{B}^{0} \rightarrow D^{0} \Lambda \bar{\Sigma}^{0}\right)}{\mathscr{B}\left(\bar{B}^{0} \rightarrow D^{0} \Lambda \bar{\Lambda}\right)}=1.5 \pm 0.9
$$

which is in better agreement with equal production of $\Lambda$ and $\Sigma^{0}$ than with the predictions of $\sim 8$ in Ref. [11].

\footnotetext{
${ }^{1}$ If we include the spin- $\frac{3}{2}$ (decuplet) baryons, we must consider $\Delta^{+} \bar{\Delta}^{-}, \Delta^{+} \bar{p}$ and $p \bar{\Delta}^{-}$for the $u \bar{u}$ pair and $\Sigma^{* 0} \bar{\Sigma}^{* 0}$, $\Sigma^{* 0} \bar{\Sigma}^{0}, \Sigma^{0} \bar{\Sigma}^{* 0}, \Sigma^{* 0} \bar{\Lambda}, \Lambda \bar{\Sigma}^{* 0}$ for the $s \bar{s}$ pair, and ignoring the suppression with respect to the spin- $\frac{1}{2}$ (octet) baryons the ratio $1: 12$ is replaced by $4: 27 \approx 1: 7$.
} 


\section{Acknowledgements}

I wish to thank many colleagues from the BABAR collaboration for useful discussions and help in preparing this talk.

\section{References}

[1] ARGUS Collab., Measurement of inclusive baryon production in B meson decays, Z. Phys. C56 (1992) 1-6.

[2] The Particle Data Group, J. Beringer et al., Phys. Rev. D86 (2012) 010001 and online update 2013.

[3] BABAR Collab., Study of the decay $\bar{B}^{0} \rightarrow \Lambda_{c}^{+} \bar{p} \pi^{+} \pi^{-}$and its intermediate states, Phys. Rev. D87 (2013) 092004

[4] Belle Collab., Study of decay mechanisms in $B^{-} \rightarrow \Lambda_{c}^{+} \bar{p} \pi^{-}$decays and observation of low-mass structure in the $\left(\Lambda_{c}^{+} \bar{p}\right)$ system, Phys. Rev. Lett. 97 (2006) 242001.

[5] BABAR Collab., Measurements of $\mathscr{B}\left(\bar{B}^{0} \rightarrow \Lambda_{c}^{+} \bar{p}\right)$ and $\mathscr{B}\left(B^{-} \rightarrow \Lambda_{c}^{+} \bar{p} \pi^{-}\right)$and studies of $\Lambda_{c}^{+} \pi^{-}$ resonances, Phys. Rev. D78 (2008) 112003.

[6] BABAR Collab., Observation and study of the baryonic B-meson decays $B \rightarrow D^{(*)} p \bar{p}(\pi)(\pi)$, Phys. Rev. D85, (2012) 092017.

[7] C.-K. Chua, W.-S. Hou, S.-Y. Tsai, Understanding $B \rightarrow D^{*-} N \bar{N}$ and its implications, Phys. Rev. D65 (2002) 034003; M. Suzuki, Partial waves of baryon-antibaryon in three-body B meson decay, J. Phys. G34 (2007) 283-298.

[8] BABAR Collab., Search for the decay $\bar{B}^{0} \rightarrow \Lambda_{c} p \bar{p} p$, SLAC-PUB-15772, to be submitted to Phys. Rev. D.

[9] BABAR Collab., Evidence for the baryonic decay $\bar{B}^{0} \rightarrow D^{0} \Lambda \bar{\Lambda}$, SLAC-PUB-15835, to be submitted to Phys. Rev. D.

[10] Yu-Kuo Hsiao, Charmful baryonic $B \rightarrow \mathscr{B} \overline{\mathscr{B}} \mathscr{M}_{c}$ decays, Int. J. Mod. Phys. A24 (2009) 3638.

[11] C.H. Chen, H.Y. Cheng, C.Q. Geng, Y.K. Hsiao, Charmful Three-body Baryonic B decays, Phys. Rev. D78 (2008) 054016. 